\title{
Assessment of Atrial Conduction Abnormalities in Patients with Hypertrophic Cardiomyopathy Before and One Year after Alcohol Septal Ablation
}

\author{
Shi Chen ${ }^{a}$ Jiansong Yuan ${ }^{a}$ Jiafen Zhang ${ }^{b}$ Shubin Qiao ${ }^{a}$ Fujian Duan $^{b}$ \\ Xiuzhang Lv ${ }^{b}$ Fenghuan $\mathrm{Hu}^{a}$ Weixian Yang ${ }^{a}$ Yuejin Yang ${ }^{a}$ \\ Departments of a Cardiology and bechocardiography, Fuwai Hospital, National Center for Cardiovascular Disease, \\ Chinese Academy of Medical Sciences and Peking Union Medical College, Beijing, China
}

\section{Key Words}

Hypertrophic cardiomyopathy · Atrial fibrillation · Atrial

electromechanical delay $\cdot$ Percutaneous transluminal

septal myocardial ablation

\begin{abstract}
Objectives: Intra- and interatrial electromechanical delay (AEMD) can be used to evaluate the development of atrial fibrillation (AF). Percutaneous transluminal septal myocardial ablation (PTSMA) is an alternative therapy for patients with hypertrophic obstructive cardiomyopathy (HOCM) that results in sustained improvements in atrial structure and function. We investigated the effects of PTSMA on the intraand inter-AEMD of HOCM patients using tissue Doppler imaging. Methods: Conventional echocardiographic and AEMD parameters were obtained in 25 healthy controls and 31 HOCM patients before and 1 year after septal ablation procedures. Results: Compared with the healthy controls, the left atrial volumes indexed by body surface area (LAVI) and the intra- and inter-AEMD were significantly higher in the HOCM patients. At 1 year after PTSMA, the LAVI was decreased (37.2 \pm 11.4 to $\left.27.0 \pm 8.5 \mathrm{ml} / \mathrm{m}^{2}, p<0.001\right)$. The intra- and inter-AEMD were also significantly decreased (22.7 \pm 9.2 to $16.6 \pm 7.7 \mathrm{~ms}, \mathrm{p}<0.001$ and $37.0 \pm 8.4$ to $26.6 \pm$ $8.0 \mathrm{~ms}, \mathrm{p}<0.001$, respectively). These changes correlated
\end{abstract}

well with the reductions in LAVI $(r=0.83, p<0.001 ; r=0.66$, $p<0.001)$. Conclusions: Both the intra- and inter-AEMD were significantly prolonged in the HOCM patients. PTSMA can improve the prolonged and inhomogeneous propagation of sinus impulses in atria.

Copyright $\odot 2012$ S. Karger AG, Basel

\section{Introduction}

In patients with hypertrophic cardiomyopathy (HCM), atrial fibrillation (AF) is the most common clinical complication, with a prevalence exceeding $20 \%$ in recent studies [1-3]. For HCM patients, AF has been associated with substantial risks of heart failure-related mortalities, strokes and severe functional disabilities [4]. Left ventricular (LV) diastolic dysfunction is an important pathophysiological feature of HCM that contributes to elevated left atrial (LA) and pulmonary vascular pressure [5]. The thin-walled left atrium is sensitive to volume and pressure changes. Sustained elevated pressure on the left atrium leads to its remodeling, which is closely associated with the occurrence of AF [6-8].

S.C. and J.Y. contributed equally to this study and share first authorship.

\section{KARGER}

Fax +41613061234 E-Mail karger@karger.ch www.karger.com (c) 2012 S. Karger AG, Basel

$0008-6312 / 12 / 1234-0254 \$ 38.00 / 0$

Accessible online at:

www.karger.com/crd
Prof. Shubin Qiao

Department of Cardiology, Cardiovascular Institute and Fuwai Hospital Peking Union Medical College and Chinese Academy of Medical Sciences No. 167 Beilishi Road, Xicheng District, Beijing (China)

E-Mail qsbmail@tom.com 
Percutaneous transluminal septal myocardial ablation (PTSMA) is an alternative therapy for patients with hypertrophic obstructive cardiomyopathy (HOCM) that results in long-term improvement in diastolic dysfunction and also partially reverses LA remodeling [9-11]. However, it is still uncertain whether PTSMA can reduce the risk of AF in HOCM patients.

The echocardiographic evaluation of electrical events, especially atrial electromechanical delay (AEMD), is novel in cardiac ultrasound practice. With recent developments in tissue Doppler imaging (TDI), it is possible to precisely assess atrial mechanical events from different regions with high temporal resolutions. AEMD can be measured from the onset of the P-wave on the electrocardiogram (ECG) to the onset of atrial contraction as determined by TDI $[12,13]$. Previous studies have proven that TDI-derived intra- and inter-AEMD values are predictors of AF $[14,15]$.

To our knowledge, AEMD has not been evaluated in HCM patients. In this study, we assessed AEMD using TDI in HOCM patients before and 1 year after septal ablation to investigate the severities of their atrial electrophysiological abnormalities and the effects of PTSMA on the intra- and inter-AEMD.

\section{Methods}

\section{Study Population}

Between January and December 2010, 45 consecutive patients with symptomatic HOCM were referred for PTSMA to our center. The diagnoses of HCM were obtained by means of 2-dimensional echocardiography in patients with interventricular septal thicknesses of $\geq 1.5 \mathrm{~cm}$ who had no other causes attributed to their LV hypertrophy. The selection criteria for PTSMA were as follows: the persistence of symptoms despite being administered the maximum tolerated dosage of medication, LV outflow tract (LVOT) gradient $>50 \mathrm{~mm} \mathrm{Hg}$ at rest or $>100 \mathrm{~mm} \mathrm{Hg}$ after provocation, accessible septal branches, typically of the left anterior descending coronary artery, the absence of a significant intrinsic abnormality of the mitral valve and other conditions for which cardiac surgery was indicated. Finally, 37 patients underwent septal ablation and 8 underwent surgical myectomy and mitral valve replacement because of serious mitral regurgitation. We excluded 6 patients due to the inability to deliver ethanol (3 cases) or incomplete echocardiographic parameters ( 3 cases) from the 37 patients who underwent PTSMA. The final cohort included 31 patients for whom the echocardiographic parameters were available at the baseline and 1-year follow-up evaluation. Twenty-five age- and gender-matched healthy controls were included from the subjects who visited our hospital for annual routine medical examinations. This study was approved by the ethics committee of the Fuwai Hospital, China.

Atrial Conduction Abnormalities in

Hypertrophic Cardiomyopathy Patients
PTSMA Procedure

A 6-French transfemoral temporary pacemaker was positioned in the right ventricle. After initial angiography to localize the origin of the septal perforating arteries, a 9-mm-long, oversized, over-the-wire balloon $(1.5-2.5 \mathrm{~mm})$ catheter was introduced over a 0.014 -inch guidewire into the septal perforator artery and inflated. Contrast medium (SonoVue, Bracco Imaging BV, Geneva, Switzerland) was then injected through the balloon catheter lumen under continuous echocardiographic imaging. Once it was confirmed that the territory that was supplied by that septal branch corresponded to the basal septum causing the LVOT obstruction and that no other myocardial territory was involved, 1-4 $\mathrm{ml}$ of ethanol was injected into the septal branch. Repeated evaluations of the presence and extent of LVOT obstruction at rest and after amyl nitrite inhalation were performed using catheters and Doppler echocardiography to ensure the success of the intervention. A successful procedure was defined as a reduction in the LVOT pressure gradient of $50 \%$ of the baseline. For patients with $<50 \%$ reduction in either the resting or provoked LVOT gradient, other septal arteries were targeted and treated in a similar fashion.

\section{Echocardiography}

Each subject underwent an echocardiographic evaluation using an iE33 echocardiographic system (Philips Medical Systems, Best, The Netherlands) that was equipped with an S5-1 transducer (frequency transmitted, $1.7 \mathrm{MHz}$; frequency received, $3.4 \mathrm{MHz}$ ) before and 1 year after the PTSMA procedure. One-lead ECG was recorded continuously. Two-dimensionally guided M-mode echocardiography was performed to measure the thicknesses of the interventricular septum and LV posterior wall (LVPW) and the diameter of the left atrium by standard methods. The LV enddiastolic and end-systolic volumes (LVEDV and LVESV), ejection fraction and LA end-systolic volume (LAESV) were calculated using a modified Simpson's biplane method in the apical 4- and 2 -chamber views. The maximal early and late diastolic inflow velocities (E and A waves), E/A ratio, and deceleration time (DT) of the E wave were obtained using pulsed-wave Doppler. The sample volume was placed just below the level of the mitral leaflet tips in the apical 4-chamber view. The LVOT gradient was measured using continuous-wave Doppler in the apical 5-chamber view. The TDI of the mitral annulus movement was performed using the apical 4-chamber view. A $1.5-\mathrm{mm}$ sample volume was placed at the lateral mitral annular sites. Analyses of the early and late diastolic peak velocities ( $\mathrm{Ea}$ and $\mathrm{Aa}$ ) and isovolumic relaxation time (IVRT) were performed. The AEMD was defined as the time interval from the onset of the P wave on the surface ECG to the beginning of the late diastolic wave (Aa wave) (fig. 1). It was measured from the lateral mitral annulus (mitral AEMD), inferoseptal mitral annulus (septum AEMD) and tricuspid annulus (tricuspid AEMD). All AEMD intervals were averaged over 3 consecutive beats. The difference between the mitral AEMD and tricuspid AEMD intervals was defined as the inter-AEMD, and the difference between the septum AEMD and tricuspid AEMD intervals was defined as the intra-AEMD.

\section{Electrocardiography}

All subjects underwent a 12-lead ECG recording after a 20min resting period in the supine position at a paper speed of 50 $\mathrm{mm} / \mathrm{s}$ and $2 \mathrm{mV} / \mathrm{cm}$ before and 1 year after PTSMA. The P-wave 
durations were measured manually in all of the simultaneously recorded 12 leads of the surface ECG by 1 observer who was unaware of the study hypothesis. The mean P-wave duration of at least 3 complexes was calculated for each lead. The onset of the $\mathrm{P}$ wave was defined as the point of the first visible upward departure from the baseline for the positive waveforms and as the point of the first downward departure from the baseline for the negative waveforms. The return to baseline was considered to be the end of the $\mathrm{P}$ wave. The $\mathrm{P}_{\max }$ that was measured in from any of the 12 leads of the surface ECG was used as the longest atrial conduction time. The difference between $\mathrm{P}_{\max }$ and $\mathrm{P}_{\min }$ was calculated and defined as $\mathrm{P}_{\mathrm{d}}$.

\section{Statistical Analyses}

The data are presented as the means \pm SD for the continuous variables or as percentages for the categorical variables. The clinical characteristics were compared using the test for the continuous variables and the $\chi^{2}$ test for the categorical variables. The correlations between changes in the intra- and inter-AEMD and the improvement of the LA volume indexed to the body surface area (LAVI) were examined using the Spearman's test. All of the probability values that were used were for 2 -tailed tests. A value of $p<0.05$ was considered to be indicative of a statistically significant result. The data processing and statistical analyses were performed using the SPSS 17.0 software (SPSS, Chicago, Ill., USA).

\section{Results}

The basic clinical characteristics of the 31 HOCM patients and 25 normal subjects are listed in table 1 . There were no significant differences between patients and healthy controls in terms of age, gender, height, weight, heart rate, systolic blood pressure and diastolic blood pressure. In patients with HOCM, the mean NYHA class was $2.6 \pm 0.7$, despite optimal medical therapy that consisted of beta-blockers in $24(77.4 \%)$ patients and calciumchannel blockers in 8 (25.8\%).

The conventional echocardiographic characteristics are shown in table 2. Compared with healthy controls, HOCM patients prior to the PTSMA procedure exhibited higher septal and LVPW thicknesses, LAESV, LAVI and LV ejection fraction, but lower LVEDV and LVESV. The transmitral E-wave velocities, $\mathrm{A}$-wave velocities and $\mathrm{E} / \mathrm{A}$ ratios were similar between the 2 groups, whereas the DT, IVRT and E/Ea ratios were higher in patients with HOCM. The lateral Ea was significantly lower in the HOCM patients. The mean baseline resting LVOT gradient for the HOCM patients was $91.7 \pm 22.4 \mathrm{~mm} \mathrm{Hg}$.

P-wave indices are shown in table 3 . The $\mathrm{P}_{\max }$ was significantly greater in the HOCM patients prior to PTSMA compared with the healthy controls. The $\mathrm{P}_{\text {min }}$ did not differ significantly between the groups. The $\mathrm{P}_{d}$ was significantly higher in the patients with HOCM. Table 4 shows

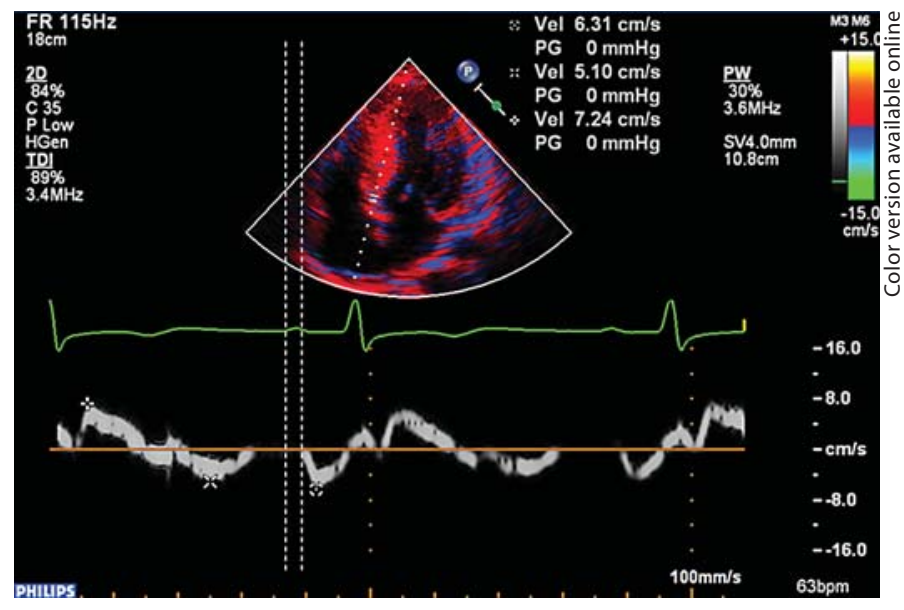

Fig. 1. Measurement of the AEMD from the onset of the $P$ wave on surface ECG to the beginning of the Aa wave with tissue Doppler echocardiography.

Table 1. Clinical characteristics of the study subjects

\begin{tabular}{|c|c|c|c|}
\hline & $\begin{array}{l}\text { Control } \\
\text { subjects } \\
(\mathrm{n}=25)\end{array}$ & $\begin{array}{l}\text { HOCM } \\
\text { patients } \\
(\mathrm{n}=31)\end{array}$ & $\begin{array}{l}\mathrm{p} \\
\text { value }\end{array}$ \\
\hline Age, years & $43.4 \pm 11.3$ & $46.1 \pm 10.8$ & 0.373 \\
\hline Male, n (\%) & $15(60.0)$ & $23(74.2)$ & 0.388 \\
\hline Height, cm & $165.5 \pm 7.1$ & $166.7 \pm 7.7$ & 0.548 \\
\hline Weight, kg & $66.0 \pm 11.1$ & $70.7 \pm 10.9$ & 0.122 \\
\hline $\begin{array}{l}\text { Family history of hypertrophic } \\
\text { cardiomyopathy, } \mathrm{n}(\%)\end{array}$ & 0 & $10(32.3)$ & - \\
\hline Previous syncope, n (\%) & 0 & $11(35.5)$ & - \\
\hline Previous dyspnea, n (\%) & 0 & $20(64.5)$ & - \\
\hline Previous angina pectoris, $\mathrm{n}(\%)$ & 0 & $10(32.3)$ & - \\
\hline Heart rate, bpm & $68.9 \pm 6.6$ & $67.7 \pm 7.4$ & 0.551 \\
\hline SBP, mm Hg & $119.2 \pm 7.6$ & $115.2 \pm 11.3$ & 0.147 \\
\hline $\mathrm{DBP}, \mathrm{mm} \mathrm{Hg}$ & $71.7 \pm 6.7$ & $72.1 \pm 11.0$ & 0.878 \\
\hline NYHA classes & - & $2.6 \pm 0.7$ & - \\
\hline \multicolumn{4}{|l|}{ Medical treatment, n (\%) } \\
\hline$\beta$-Blocker & 0 & $24(77.4)$ & - \\
\hline Calcium-channel blocker & 0 & $8(25.8)$ & - \\
\hline
\end{tabular}

DBP $=$ Diastolic blood pressure; NYHA = New York Heart Association; SBP $=$ systolic blood pressure.

the TDI-derived AEMD parameters. Compared with the controls, the AEMD values that were measured at the lateral mitral annulus, inferoseptal mitral annulus, and tricuspid annulus were significantly prolonged in the HOCM patients prior to PTSMA and both the intra- and inter-AEMD were significantly higher $(22.7 \pm 9.2$ vs. 
Table 2. Conventional echocardiographic characteristics of the healthy controls and HOCM patients at baseline and 1 year after PTSMA

\begin{tabular}{lccc}
\hline Characteristics & $\begin{array}{l}\text { Healthy } \\
\text { controls } \\
(\mathrm{n}=25)\end{array}$ & $\begin{array}{l}\text { HOCM } \\
\text { patients } \\
\text { at baseline } \\
(\mathrm{n}=31)\end{array}$ & $\begin{array}{l}\text { HOCM } \\
\text { patients 1 year } \\
\text { after PTSMA } \\
(\mathrm{n}=31)\end{array}$ \\
\hline Septum thickness, mm & $8.4 \pm 1.3$ & $19.6 \pm 4.7^{\mathrm{c}}$ & $16.5 \pm 4.3^{\mathrm{c}, *}$ \\
LVPW thickness, mm & $8.5 \pm 1.5$ & $12.6 \pm 3.4^{\mathrm{c}}$ & $11.7 \pm 2.4^{\mathrm{c}}$ \\
LA diameters, mm & $31.3 \pm 3.2$ & $40.8 \pm 9.2^{\mathrm{c}}$ & $36.9 \pm 6.2^{\mathrm{c}, \#}$ \\
LAESV, ml & $34.6 \pm 8.6$ & $68.4 \pm 21.3^{\mathrm{c}}$ & $49.2 \pm 14.8^{\mathrm{c}, \S}$ \\
LAV index, ml/m ${ }^{2}$ & $19.5 \pm 4.2$ & $37.2 \pm 11.4^{\mathrm{c}}$ & $27.0 \pm 8.5^{\mathrm{c}, \S}$ \\
LVEDV, ml & $88.0 \pm 20.3$ & $76.9 \pm 17.7^{\mathrm{a}}$ & $82.5 \pm 12.1^{\#}$ \\
LVESV, ml & $32.7 \pm 12.6$ & $18.4 \pm 6.9^{\mathrm{c}}$ & $24.6 \pm 5.0^{\mathrm{b}, \S}$ \\
LVEF, \% & $64.6 \pm 5.0$ & $77.4 \pm 6.6^{\mathrm{c}}$ & $77.5 \pm 6.0^{\mathrm{c}}$ \\
LVOT gradient, mm Hg & 0 & $91.7 \pm 22.4^{\mathrm{c}}$ & $27.1 \pm 15.4^{\mathrm{c}, \S}$ \\
Mitral E velocity, cm/s & $83.1 \pm 15.2$ & $83.8 \pm 24.7$ & $72.9 \pm 18.4^{\mathrm{a}}$ \\
Mitral A velocity, cm/s & $65.1 \pm 20.1$ & $81.0 \pm 34.5^{\mathrm{a}}$ & $81.2 \pm 32.3^{\mathrm{a}}$ \\
E/A ratio & $1.4 \pm 0.6$ & $1.2 \pm 0.6$ & $1.0 \pm 0.5^{\mathrm{a}}$ \\
E deceleration time, ms & $174.6 \pm 8.9$ & $226.6 \pm 11.7^{\mathrm{c}}$ & $192.5 \pm 10.1^{\mathrm{c}, \S}$ \\
IVRT, ms & $76.6 \pm 6.8$ & $122.8 \pm 8.6^{\mathrm{c}}$ & $96.2 \pm 8.0^{\mathrm{c}, \S}$ \\
Lateral Ea, cm/s & $11.9 \pm 3.8$ & $6.0 \pm 1.9^{\mathrm{c}}$ & $7.2 \pm 1.8^{\mathrm{c}, *}$ \\
Lateral Aa, cm/s & $8.4 \pm 2.4$ & $7.5 \pm 2.6$ & $9.6 \pm 3.0^{*}$ \\
E/Ea ratio & $7.6 \pm 2.5$ & $15.1 \pm 5.4^{\mathrm{c}}$ & $10.9 \pm 4.4^{\mathrm{b}, *}$ \\
\hline & & &
\end{tabular}

$\mathrm{LA}=$ Left atrium; $\mathrm{LVEF}=$ left ventricular ejection fraction.

${ }^{\mathrm{a}} \mathrm{p}<0.05$ vs. control subjects; ${ }^{\mathrm{b}} \mathrm{p}<0.01$ vs. control subjects; ${ }^{\mathrm{c}} \mathrm{p}<0.001$ vs. control subjects; ${ }^{\#} \mathrm{p}<0.05$ vs. HOCM patients at baseline; ${ }^{*} \mathrm{p}<0.01 \mathrm{vs}$. HOCM patients at baseline; ${ }^{\S} \mathrm{p}<0.001$ vs. HOCM patients at baseline.

Table 3. Electrocardiographic variables of the healthy controls and HOCM patients at baseline and 1 year after PTSMA

\begin{tabular}{llll}
\hline & $\begin{array}{l}\text { Healthy } \\
\text { controls } \\
(\mathrm{n}=25)\end{array}$ & $\begin{array}{l}\text { HOCM patients } \\
\text { at baseline } \\
(\mathrm{n}=31)\end{array}$ & $\begin{array}{l}\text { HOCM patients 1 } \\
\text { year after PTSMA } \\
(\mathrm{n}=31)\end{array}$ \\
\hline $\mathrm{P}_{\max }, \mathrm{ms}$ & $94.4 \pm 7.5$ & $116.9 \pm 7.0^{*}$ & $105.0 \pm 6.4^{*, \S}$ \\
$\mathrm{P}_{\min }, \mathrm{s}$ & $63.6 \pm 4.0$ & $65.8 \pm 5.5$ & $64.5 \pm 6.8$ \\
$\mathrm{P}_{\mathrm{d}}, \mathrm{ms}$ & $30.8 \pm 5.6$ & $51.1 \pm 6.9^{*}$ & $40.5 \pm 7.0^{*, \S}$ \\
\hline
\end{tabular}

${ }^{*} \mathrm{p}<0.001$ vs. healthy controls; ${ }^{\S} \mathrm{p}<0.001$ vs. HOCM patients at baseline.

$12.3 \pm 6.0, \mathrm{p}<0.001$ and $37.0 \pm 8.4$ vs. $21.1 \pm 5.9, \mathrm{p}<$ 0.001 , respectively).

During the ablation procedure, the mean amount of alcohol that was injected was $2.49 \pm 0.78 \mathrm{ml}$. In 24 patients, only 1 septal branch was treated and in the other 7 patients, 2 septal branches were treated. Right-bundle branch blocks occurred at a rate of 51.6\% (16 patients). Transitory trifascicular blocks occurred at a rate of $41.9 \%$ (13 patients). No patient underwent permanent pacemaker implantation following the procedure. There was no peri-interventional mortality during the observational period.

Changes in the conventional echocardiographic parameters in the patients with HOCM at the 1-year followup are shown in table 2. The LVOT gradients decreased from $91.7 \pm 22.4$ to $27.1 \pm 15.4 \mathrm{~mm} \mathrm{Hg}$ following PTSMA. Both the LAESV $(68.4 \pm 21.3$ to $49.2 \pm 14.8 \mathrm{ml}$, $\mathrm{p}<0.001)$ and LAVI $\left(37.2 \pm 11.4\right.$ to $27.0 \pm 8.5 \mathrm{ml} / \mathrm{m}^{2}$, $\mathrm{p}<0.001)$ were significantly reduced at 1 year after PTSMA. There were no significant changes in E-wave velocity, A-wave velocity or the E/A ratio. Both the DT and IVRT were significantly shorter at the 1-year follow-up after septal ablation. Moreover, the lateral Ea and E/Ea ratio significantly improved following PTSMA. However, the diastolic function indexes including LAVI, E/A ratio, DT, IVRT and E/Ea ratio were still abnormal compared with the control group.

Although $\mathrm{P}$-wave indices and TDI-derived AEMD were still longer than those in healthy controls, they were significantly improved after septal ablation. Table 3 and table 4 list detail parameters. There was no significant change in $\mathrm{P}_{\min }$, but $\mathrm{P}_{\max }$ and $\mathrm{P}_{\mathrm{d}}$ decreased significantly $(116.9 \pm 7.0$ to $105.0 \pm 6.4 \mathrm{~ms}, \mathrm{p}<0.001$ and $51.1 \pm 6.9$ to $40.5 \pm 7.0 \mathrm{~ms}, \mathrm{p}<0.001$, respectively) at 1 year after PTSMA.PTSMA significantly decreased all of the AEMD parameters in the patients (mitral AEMD $76.9 \pm 11.5$ to $64.0 \pm 8.9 \mathrm{~ms}, \mathrm{p}<0.001$; septum AEMD $54.1 \pm 5.2$ to $47.4 \pm 3.5 \mathrm{~ms}, \mathrm{p}<0.001$; tricuspid AEMD $39.8 \pm 7.6$ to $37.4 \pm 5.3 \mathrm{~ms}, \mathrm{p}=0.046$ ). Both the intra- and interAEMD were significantly shorter at 1 year after septal ablation (22.7 \pm 9.2 to $16.6 \pm 7.7 \mathrm{~ms}, \mathrm{p}<0.001$ and $37.0 \pm 8.4$ to $26.6 \pm 8.0 \mathrm{~ms}, \mathrm{p}<0.001$, respectively). Moreover, the changes in the intra- and inter-AEMD correlated well with the reductions in LAVI $(\mathrm{r}=0.83, \mathrm{p}<$ 0.001 , fig. $2 a ; r=0.66, p<0.001$, fig. $2 b$ ).

\section{Discussion}

Our study provides unprecedented data regarding the AEMD parameters in HOCM patients before and 1 year after the PTSMA procedure. We found that compared with healthy controls, the AEMD measurements of different sites by TDI were significantly prolonged in the HOCM patients. Moreover, both the intra- and interAEMD were significantly higher. At 1 year after septal 
Fig. 2. The relationship between the reductions in the LAVI and the changes in the AEMD parameters in an HOCM patient at 1 year after successful septal ablation. a The correlation of the changes in the intra-AEMD with the changes in the LAVI. b The correlation of the changes in the inter-AEMD with the changes in the LAVI.

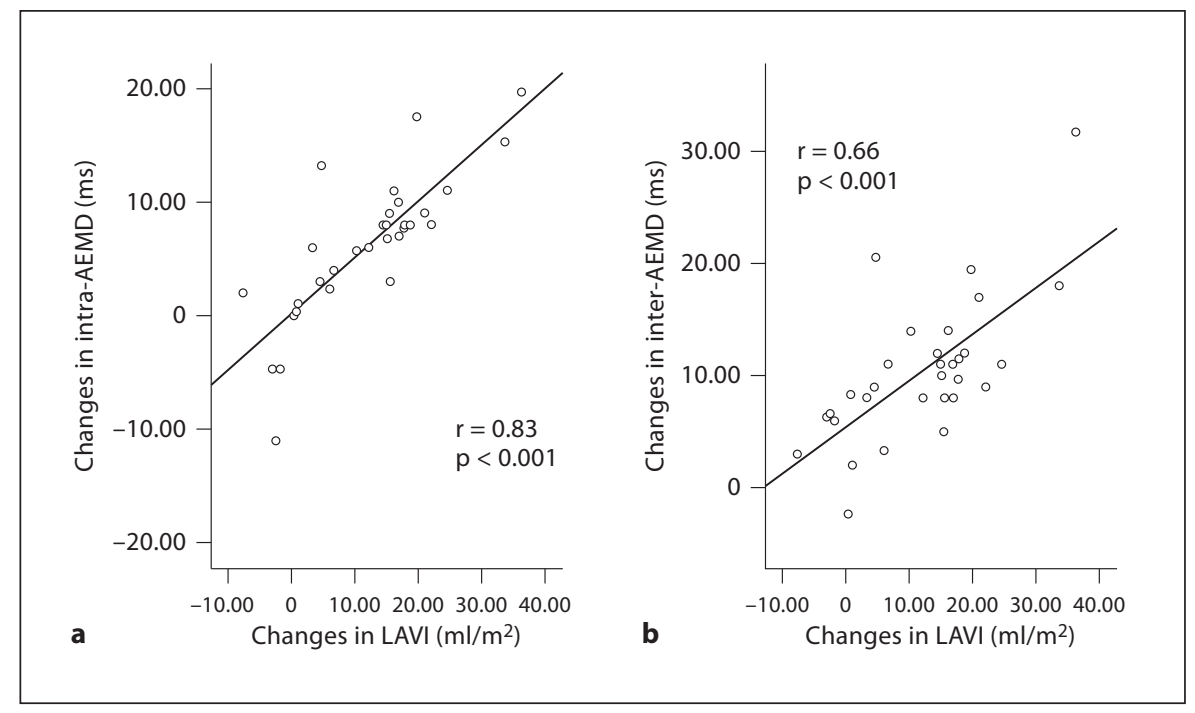

Table 4. AEMD of the healthy controls and HOCM patients at baseline and 1 year after PTSMA

\begin{tabular}{llll}
\hline & $\begin{array}{l}\text { Healthy } \\
\text { controls } \\
(\mathrm{n}=25)\end{array}$ & $\begin{array}{l}\text { HOCM patients } \\
\text { at baseline } \\
(\mathrm{n}=31)\end{array}$ & $\begin{array}{l}\text { HOCM patients } \\
\text { 1 year after } \\
\text { PTSMA } \\
(\mathrm{n}=31)\end{array}$ \\
\hline Mitral AEMD, ms & $51.7 \pm 7.4$ & $76.9 \pm 11.5^{\mathrm{c}}$ & $64.0 \pm 8.9^{\mathrm{c}, \S}$ \\
Septum AEMD, ms & $39.4 \pm 6.3$ & $54.1 \pm 5.2^{\mathrm{c}}$ & $47.4 \pm 3.5^{\mathrm{c}, \S}$ \\
Tricuspid AEMD, ms & $30.6 \pm 5.4$ & $39.8 \pm 7.6^{\mathrm{c}}$ & $37.4 \pm 5.3^{\mathrm{c}, \#}$ \\
Intra-AEMD, ms & $12.3 \pm 6.0$ & $22.7 \pm 9.2^{\mathrm{c}}$ & $16.6 \pm 7.7^{\mathrm{a}, \S}$ \\
Inter-AEMD, ms & $21.1 \pm 5.9$ & $37.0 \pm 8.4^{\mathrm{c}}$ & $26.6 \pm 8.0^{\mathrm{b}, \S}$ \\
\hline
\end{tabular}

${ }^{\mathrm{a}} \mathrm{p}<0.05$ vs. healthy controls; ${ }^{\mathrm{b}} \mathrm{p}<0.01$ vs. healthy controls; ${ }^{\mathrm{c}} \mathrm{p}<0.001$ vs. healthy controls; ${ }^{\#} \mathrm{p}<0.01$ vs. HOCM patients at baseline; ${ }^{\S} \mathrm{p}<0.001$ vs. HOCM patients at baseline.

ablation, all of the AEMD parameters, including the intra- and inter-AEMD, had improved significantly.

$\mathrm{AF}$ is the most common sustained arrhythmia in patients with $\mathrm{HCM}$. The recently reported prevalence of AF in several HCM populations ranged from 21 to $28 \%$ [13]. A previous study reported that HCM patients have a 4- to 6-fold increased risk of developing AF compared to the general population [2]. AF is an important cause of symptoms, morbidity and even mortality in patients with HCM. For these patients, the loss of the atrial systolic contribution to ventricular filling during AF may lead to clinical deterioration, involving progressive cardiac failure and systemic thromboembolism. Once AF has developed, the risk of ischemic stroke may increase 8 -fold in HCM patients relative to those with sinus rhythm, presumably via an increased risk of cardioembolic stroke [4].

The main pathophysiological feature of HCM is diastolic dysfunction due to abnormal relaxation and decreased chamber compliance. Abnormal diastolic functioning results in elevated LV filling pressures that are transmitted back to the left atrium [5]. The thin-walled atrium is seriously affected by increased load, and the elevated LA pressures result in an increase in LA wall tension and enlargement of the LA [6]. Mild LA enlargement is common in HCM. Consistent with previous studies, in our study, the mean LAVI in the HOCM patients was much higher than that in the healthy controls. LA enlargement has been regarded as a sensitive and specific predictor of the occurrence of AF in HCM patients [7, 8]; it can affect atrial conduction time and may be closely related to atrial electrophysiological abnormalities $[16,17]$. A previous study reported that not only structure remodeling but also electrical remodeling developed in the atria of patients with HCM [18]. Prolonged intra- and inter-AEMD and inhomogeneous propagation of sinus impulses are the major electrophysiological findings that predispose a patient to developing AF $[19,20]$. TDI-derived AEMD is a new parameter for the assessment of atrial conduction abnormalities. AEMD has been evaluated by this method in some cardiac disorders. Ozer et al. [14] evaluated the intra- and inter-AEMD in patients with mitral stenosis and reported that the inter-AEMD was longer and correlated with 
P-wave dispersion, which is known to be a marker of AF. Cui et al. [15] proved that the prolongation of the intraand inter-AEMD with TDI is an electrophysiological characteristic of atria that are prone to fibrillation. In this study, we demonstrated that both the intra- and inter-AEMD were significantly longer in HOCM patients than in healthy controls, indicating the existence of atrial electrophysiological remodeling and possibly predicting the development of new-onset AF in HOCM patients.

LVOT obstruction is present in up to two thirds of patients with HCM. Over the past 17 years, PTSMA has become an alternative to surgical myectomy to relieve LVOT obstruction and has been the preferred therapy since it was first reported in 1995 [21]. LV global diastolic function has been shown to improve immediately following a successful septal ablation. In addition, a long-term effect of PTSMA, involving the persistent improvement of global LV diastolic functioning for up to several years following the procedure, has been demonstrated $[9,10]$. In this study, the DT, IVRT and E/Ea ratios significantly improved in the HOCM patients following PTSMA, again reflecting the improved diastolic function resulting from the procedure. Septal ablation has been previously shown to result in the decrement of LA dimensions using 2-dimensional echocardiography. Hage et al. [11] assessed the effects of PTSMA on LA volume and the LA ejection fraction using 3D echocardiography. They found that the LA volume was significantly reduced and the LA ejection fraction significantly increased in HOCM patients following septal ablation. Our data were consistent with the results of previous studies. In the HOCM patients, the LAVI was significantly reduced at 1 year after PTSMA. These data confirm that PTSMA can partially reverse atrial structure remodeling and improve atrial function. John et al. [22] studied 21 patients with mitral stenosis undergoing mitral commissurotomies. Immediately following these procedures, LA volumes were reduced and the atrial electrophysiological and electroanatomical abnormalities improved consistently. This observation suggests that relieving the atrial stretch may reverse the AF substrate. However, it is unclear whether the improvement in atrial structure and function following PTSMA can reduce the risk of $\mathrm{AF}$ or the prevalence of paroxysmal AF in HOCM patients. Recently, Hosokawa et al. [23] reported on $3 \mathrm{HOCM}$ patients with refractory paroxysmal AF. Following PTSMA, the incidence of episodes of paroxysmal AF became significantly less frequent. Because there were only 3 participants in their study, we could not obtain a validated conclusion. In our study, we found that both the intra- and inter-AEMD were significantly shorter in HOCM patients following PTSMA. Our results may indicate improved impulse conduction and less electrical heterogeneity in the atria following the septal ablation. This study indirectly supports the idea that PTSMA may reduce the incidence of AF in patients with HOCM. Moreover, we found that the decrements of the intra- and inter-AEMD correlated well with the reduced LAVI values. Therefore, we can speculate that LA structural reverse remodeling may reduce the arrhythmogenic substrate, ultimately resulting in atrial electrophysiological reverse remodeling in HOCM patients following PTSMA.

Our study has several limitations. First, the study population size was small. Second, changes in atrial conduction time were assessed before and 1 year after septal ablation, but patients were not followed up for arrhythmic episodes. We could not compare the incidence of AF in HOCM patients who underwent PTSMA with that in HOCM patients who do not receive septal ablation. Therefore, our findings should be interpreted with caution. Third, in this study, we used a modified Simpson's biplane method based on 2-dimensional but not 3-dimensional echocardiography to measure the LA volume. Therefore, a large, prospective study of extended duration is necessary to confirm the effects of PTSMA on the primary prevention of AF in HOCM patients.

In conclusion, compared to the healthy controls, the intra- and inter-AEMD were significantly prolonged in the HOCM patients prior to septal ablation. PTSMA can improve upon LV diastolic dysfunction and partially reverse LA remodeling. Both the intra- and inter-AEMD were significantly reduced in the HOCM patients following PTSMA. The reduced intra- and inter-AEMD values correlated well with the changes in LAVI. Whether these reductions in the intra- and inter-AEMD reflect the decreased incidence of AF in the long-term requires further study.

\section{Conflict of Interest}

All authors have no conflicts of interest to declare. 


\section{References}

-1 Tani T, Yagi T, Kitai T, Kim K, Nakamura H, Konda T, Fujii Y, Kawai J, Kobori A, Ehara N, Kinoshita M, Kaji S, Yamamuro A, Morioka S, Kita T, Furukawa Y: Left atrial volume predicts adverse cardiac and cerebrovascular events in patients with hypertrophic cardiomyopathy. Cardiovasc Ultrasound 2011;9: 34.

2 Maron BJ, Olivotto I, Bellone P, Conte MR, Cecchi F, Flygenring BP, Casey SA, Gohman TE, Bongioanni S, Spirito P: Clinical profile of stroke in 900 patients with hypertrophic cardiomyopathy. J Am Coll Cardiol 2002;39: 301-307.

$\checkmark 3$ Kubo T, Kitaoka H, Okawa M, Hirota T, Hayato K, Yamasaki N, Matsumura Y, Yabe T, Takata J, Doi YL: Clinical impact of atrial fibrillation in patients with hypertrophic cardiomyopathy. Results from Kochi RYOMA Study. Circ J 2009;73:1599-1605.

$\checkmark 4$ Olivotto I, Cecchi F, Casey SA, Dolara A, Traverse JH, Maron BJ: Impact of atrial fibrillation on the clinical course of hypertrophic cardiomyopathy. Circulation 2001;104: 2517-2524.

5 Geske JB, Sorajja P, Nishimura RA, Ommen SR: Evaluation of left ventricular filling pressures by Doppler echocardiography in patients with hypertrophic cardiomyopathy: correlation with direct left atrial pressure measurement at cardiac catheterization. Circulation 2007;116:2702-2708.

6 Hsiao SH, Lin KL, Chiou KR: Comparison of left atrial volume parameters in detecting left ventricular diastolic dysfunction versus tissue Doppler recordings. Am J Cardiol 2012;109:748-755.

7 Pujadas S, Vidal-Perez R, Hidalgo A, Leta R, Carreras F, Barros A, Bayes-Genis A, Subirana MT, Pons-Llado G: Correlation between myocardial fibrosis and the occurrence of atrial fibrillation in hypertrophic cardiomyopathy: a cardiac magnetic resonance imaging study. Eur J Radiol 2010; 75:e88-e91.
8 Tani T, Tanabe K, Ono M, Yamaguchi K, Okada M, Sumida T, Konda T, Fujii Y, Kawa J, Yagi T, Sato M, Ibuki M, Katayama M, Tamita K, Yamabe K, Yamamuro A, Nagai K, Shiratori K, Morioka S: Left atrial volume and the risk of paroxysmal atrial fibrillation in patients with hypertrophic cardiomyopathy. J Am Soc Echocardiogr 2004;17:644648.

$\checkmark 9$ Meliga E, Steendijk P, Valgimigli M, Ten Cate FJ, Serruys PW: Effects of percutaneous transluminal septal myocardial ablation for obstructive hypertrophic cardiomyopathy on systolic and diastolic left ventricular function assessed by pressure-volume loops. Am J Cardiol 2008;101:1179-1184.

10 Jassal DS, Neilan TG, Fifer MA, Palacios IF Lowry PA, Vlahakes GJ, Picard MH, Yoerger DM: Sustained improvement in left ventricular diastolic function after alcohol septa ablation forhypertrophic obstructive cardiomyopathy. Eur Heart J 2006;27:1805-1810.

11 Hage FG, Karakus G, Luke WD Jr, Suwanjutah T, Burri MV, Nanda NC, Aqel RA: Effect of alcohol-induced septal ablation on left atrial volume and ejection fraction assessed by real time three-dimensional transthoracic echocardiography in patients with hypertrophic cardiomyopathy. Echocardiography 2008;25:784-789.

12 Yavuz B, Deniz A, Ertugrul DT, Deveci OS, Yalcin AA, Ata N, Kucukazman M, Dal K, Tutal E: A novel echocardiographic marker in hypertensive patients: is diastolic dysfunction associated with atrial electromechanical abnormalities in hypertension? J Clin Hypertens (Greenwich) 2010;12:687692.

13 Sengul C, Cevik C, Ozveren O, Oduncu V, Sunbul A, Kilıcarslan F, Semiz E, Dindar I: Telmisartan decreases atrial electromechanical delay in patients with newly diagnosed essential hypertension. J Electrocardiol 2012;45:123-128.

-14 Ozer N, Yavuz B, Can I, Atalar E, Aksöyek S, Ovünç K, Ozmen F, Kes S: Doppler tissue evaluation of intra-atrial and interatrial electromechanical delay and comparison with P-wave dispersion in patients with mitral stenosis. J Am Soc Echocardiogr 2005; 18: 945-948.

-15 Cui QQ, Zhang W, Wang H, Sun X, Wang R, Yang HY, Meng XQ, Zhang Y, Wang H: Assessment of atrial electromechanical coupling and influential factors in nonrheumatic paroxysmal atrial fibrillation. Clin Cardiol 2008;31:74-78.
16 Kim SJ, Choisy SC, Barman P, Zhang H, Hancox JC, Jones SA, James AF: Atrial remodeling and the substrate for atrial fibrillation in rat hearts with elevated afterload. Circ Arrhythm Electrophysiol 2011;4:761769.

17 Lau DH, Mackenzie L, Kelly DJ, Psaltis PJ, Brooks AG, Worthington M, Rajendram A, Kelly DR, Zhang Y, Kuklik P, Nelson AJ, Wong CX, Worthley SG, Rao M, Faull RJ, Edwards J, Saint DA, Sanders P: Hypertension and atrial fibrillation: evidence of progressive atrial remodeling with electrostructural correlate in a conscious chronically instrumented ovine model. Heart Rhythm 2010;7: 1282-1290.

18 Ozdemir O, Soylu M, Demir AD, Topaloglu S, Alyan O, Turhan H, Bicer A, Kutuk E: Pwave durations as a predictor for atrial fibrillation development in patients with hypertrophic cardiomyopathy. Int J Cardiol 2004; 94:163-166.

19 Pytkowski M, Jankowska A, Maciag A, Kowalik I, Sterlinski M, Szwed H, Saumarez RC: Paroxysmal atrial fibrillation is associated with increased intra-atrial conduction delay. Europace 2008;10:1415-1420.

-20 Xia Y, Hertervig E, Kongstad O, Ljungström E, Platonov P, Holm M, Olsson B, Yuan S: Deterioration of interatrial conduction in patients with paroxysmal atrial fibrillation: electroanatomic mapping of the right atrium and coronary sinus. Heart Rhythm 2004; 1: 548-553.

21 Sigwart U: Non-surgical myocardial reduction for hypertrophic obstructive cardiomyopathy. Lancet 1995;346:211-214.

22 John B, Stiles MK, Kuklik P, Brooks AG, Chandy ST, Kalman JM, Sanders P: Reverse remodeling of the atria after treatment of chronic stretch in humans: implications for the atrial fibrillation substrate. J Am Coll Cardiol 2010;55:1217-1226.

-23 Hosokawa Y, Takano H, Ohno T, Takayama M, Takano T: Impact of percutaneous transluminal septal myocardial ablation on refractory paroxysmal atrial fibrillation in patients with hypertrophic obstructive cardiomyopathy. Angiology 2008;59:329-334. 